\title{
DOCUMENTACIÓN DIGITAL Y LÉXICO EN LA TRADUCCIÓN E INTERPRETACIÓN EN LOS SERVICIOS PÚBLICOS (TISP): FUNDAMENTOS TEÓRICOS Y PRÁCTICOS \\ María del Mar Sánchez Ramos \\ Berlin: Peter Lang, 2020
}

\section{AGNIESZKA BIERNACKA \\ University of Warsaw, Poland \\ a.biernacka@uw.edu.pl}

Public service interpretation and translation (PSIT) has been attracting more and more attention as a field of study in the last few decades. This has contributed to a growing amount of scholarly research (cf. Hale 2007; Mikkelson 2017) and the creation of programmes within higher education studies aimed at preparing informed professionals (Valero-Garcés 2019). The book submitted for review is about digital documentation for the purposes of PSIT, representing a useful addition to the literature on the latest trends within the field. The topic falls within the framework of translation and interpreting in various settings where two languages, two cultures and two different viewpoints meet and interact. It relates to new technologies used by public service interpreters and translators as tools for creating glossaries and advanced lexical databases as well as for post-editing machine translation.

The main body of the publication is divided into four chapters, preceded by an introduction and followed by a conclusions section. Moreover, the book contains references, an index of charts (179) and an index of tables (181), which help organize the text and navigate its content.

In the introduction, the author underlines the importance of public service translation and interpreting and of raising awareness among (prospective) practitioners of the successive stages of the translation and interpreting process. Moreover, the introduction includes short descriptions of the content of the 


\section{Reviews}

individual chapters and, interestingly, a separate paragraph concerning the bibliography in which the author justifies the selection of publications included in her book. Such a justification could be a regular component of monographs, whose authors cannot carry out an all-encompassing and updated literature review on the topic in question, due to the mere impossibility of reviewing all previously published sources and because of the sometimes long publishing process. In addition, it has the potential to act as an apology to those scholars who might feel offended by not being included in an extensive literature review.

Chapter 1 is a literature review relating to concepts and definitions of PSIT, with special attention paid to its development and evolution within university studies in Spain. The author lists the following features of PSIT as distinct from other modalities of interpreting and translation: a knowledge of both source and target language and culture, the scarce recognition of the profession, and the fact that it exceeds the borders of mere translation and/or interpreting by assuming responsibility for coordinating the transfer between two languages and two cultures. Although the author focuses on the situation of PSIT in Spain, the three above-mentioned characteristics are universal. For example, in Poland the recognition of the profession in terms of gaining a position and reputation in the market is still low despite the existence of the Act on the Profession of the Sworn Translator of 25 November 2004, which is binding upon PSIT practitioners who render their services for the courts, public prosecutors, the police and public administration bodies (cf. Biernacka 2019: 41-42). Chapter 1 also discusses a teaching programme successfully implemented at the University of Alcalá, Spain, aimed at preparing specialists in the field of PSIT. Due to the fact that training for the purposes of PSIT is of vital importance for many countries worldwide, a detailed description of the original programme can be appreciated not only from a theoretical but also from a practical point of view. In other words, by including this information, the author both satisfies the needs of researchers in PSIT involved in an academic discussion of learning outcomes (knowledge, skills and attitudes) included in such programmes and provides guidelines for other universities which may now be considering the introduction of a similar training programme.

The aim of Chapter 2 is to contextualize the lexis within translator competence models. The author points out that linguistic and documentary needs are of prime importance among those reported by public service interpreters and translators (27) and this is a point of departure for the study as a whole presented in the book. To support this statement, the author elaborates on various approaches to a definition of translation competence and, in particular, on a communicative approach as occupying an important place in PSIT. She then moves on to discuss different models of translation competence. Finally, and most importantly, the 


\section{Reviews}

author indicates that technology is, according to the EMT Competence Framework (2017: 4), one of the five main areas of translation competence. This last part is particularly interesting. It confirms that the approach adopted by PSIT training programmes should rely on the assumption that the candidates for the profession, their trainers, as well as the practitioners, are fully computer-literate and that they are capable of using new technologies to retrieve information, create digital documentation and translate texts.

Chapter 3 highlights the importance of lexical competence in the teaching of translation. This provides food for thought for public service interpreter and translator teachers who may wrongly assume that the knowledge of technical terms comes in passing and naturally. The author shows that the contrary is true. First, it is stated that the lexis used for PSIT purposes can be considered within such fields of studies as discourse analysis and second language learning. Afterwards, the author discusses translation teaching models and the way they address the lexis. The lexis is considered as one of the stages of approaching the source text, as a means to discover the social condition of the interlocutor, as an element of knowledge of the target culture and as one of the translator's general objectives (87). Finally, the author presents and discusses her proposal for the development of lexical competence in PSIT, which comprises three components: a knowledge of the structure and functioning of lexical systems in both the source and target languages, a knowledge of the use of these systems for the purposes of text comprehension and production, and a knowledge of mechanisms of adapting the lexis to the cultural context of the source and target languages (103). The main objective of the model is to facilitate and enhance the learning of the lexis within PSIT academic programmes.

Chapter 4 discusses the use of electronic dictionaries, corpora, matching programmes, machine translation and post-editing tools. This is based on the assumption that, as stated in the EMT Competence Framework, the ability to "[e]valuate the relevance and reliability of information sources" (2017: 8) and to then correctly classify and archive documents is key to interpreting and translation processes. Furthermore, the author rightly observes that the information obtained from documents is of linguistic (i.e. terminological and phraseological), thematic (specialized), textual (related to the existence of a variety of texts with which the translator copes) and cultural (communicative) character (118-119). In addition, the author lists the competences which a public service translator and interpreter should possess. It would have been relevant to discuss these competences against the background of earlier sets of competences in PSIT, for example the one proposed by Hertog (2016). Furthermore, the author indicates the quality of translation as being the objective pursued by public service interpreters and 


\section{Reviews}

translators. The chapter ends with a test verifying the quality of machine translations from English into Spanish of a medical text made with the free DeepL and SYSTRANet online translators (153-155). The source text and the two target texts are transparently presented, but the lack of a comparative analysis of these three texts by the author is disappointing. Presumably, the author intended to only give an idea of how the two tools work; however, leaving the reader without any explanation is somewhat unsatisfactory and creates a need for resorting to other - non-academic - sources which offer similar presentations accompanied by expert opinions on the quality of the translation (cf. Rubio 2020).

The conclusions are very short and comprise a review of the main issues discussed in the book. They refer back to the main aim of the book, which, in general, is to shed light on the necessity of including new technologies in PSIT in terms of training candidates for the profession and its practice by interpreters and translators. The references comprise 289 items published between 1961 and 2019. The list is impressively extensive, and the publication can thus be considered as a serious source of knowledge of PSIT and a reference point for further research in the field.

To sum up, the book can be recommended as a source of knowledge about PSIT and the potential of new technologies to serve as useful tools for public service interpreters and translators. However, the publication is not free of minor shortcomings. One of them is the lack of a clearly identifiable empirical part which, based on an analysis of particular examples, could have shown in what way the new technologies presented in Chapter 4 contribute to the digitalization of documentation for the purposes of PSIT. This would have been of particular interest not only for scholars but also for prospective users of digital documentation tools. Another drawback is that the author emphasizes translation while interpreting is referred to only because PSIT covers both. As far as the strengths are concerned, the book includes a solid and comprehensive literature review on PSIT and thus can be a reference in itself. Moreover, it certainly contributes to the development of research into PSIT in that it presents this particular type of translation and interpreting as an object of interest for new technologies, which are usually considered as tools applied in specialized (but not public service) translation and conference interpreting. In other words, the author demystifies digital documentation and successfully shows that public service interpreters and translators have the potential to be rightful users of such tools as digital databases, corpora, machine translation and post-editing. Furthermore, apart from the valuable content offering a meticulous literature review and indicating the importance of digitalization of the work of public service interpreters and translators, the language of the publication needs to be stressed; the book is written in Spanish, which is added value. In a world where a large majority of 


\section{Reviews}

academic writings are published in English as a lingua franca, it is a real scholarly fiesta to come up with such an important book in a different language. In addition, from this reviewer's point of view, whose mother tongue is Polish, it is an important issue not only because of the author's obvious contribution to spreading knowledge in a language other than English but also for technical reasons. The scholars involved in pedagogical activities are now able to refer their students - with Spanish as a second or third language - to such publications also as a source of language input of the highest possible quality. And last but not least, I would like to express my own words of appreciation as a sworn translator and interpreter, conference interpreter, and legal translator of Spanish, as well as to emphasize, on behalf of the translation and interpreting community, that we look forward to further scholarly research in this field. All in all, the book is a useful source of knowledge for various stakeholders: scholars researching the field of PSIT, students willing to explore past, present and future perspectives of PSIT as a field of study, translators and interpreters who would like to catch up with state-of-the-art knowledge of new technologies as applied to PSIT, as well as university decisionmakers interested in introducing PSIT as a necessary teaching programme reflecting current social and academic trends.

\section{Works Cited}

BieRnACKA, Agnieszka. 2019. InterpreterMediated Interactions of the Courtroom: $A$ Naturally Occurring Data Based Study. Berlin: Peter Lang.

“EMT Competence Framework". 2017. <https:// ec.europa.eu/info/sites/info/files/emt_ competence_fwk_2017_en_web.pdf $>$. Accessed July 23, 2020.

Hale, Sandra Beatriz. 2007. Community Interpreting. Houndmills, Basingstoke: Palgrave Macmillan.

Hertog, Erik. 2016. "Legal Interpreting Competences in Civil Justice and Mediation". In Townsley, Brooke (ed.) Understanding Justice: An Enquiry into Interpreting in Civil Justice and Mediation. London: Middlesex University: 51-89.
MiKKELSON, Holly. 2017. Introduction to Court Interpreting. $2^{\text {nd }}$ edition. Abingdon: Routledge.

RuBıo, Isabel. 2020. “¿Cuál es el mejor traductor?: probamos DeepL, Google Translate y Bing". El País May 30, 2020. <https://elpais.com/tecnologia/2020-05-29/ cual-es-el-mejor-traductor-probamos-deeplgoogle-translate-y-bing.html>. Accessed May $30,2020$.

VAlero-Garcés, Carmen. 2019. “Navigating between Theory and Practice: Design and Implementation of a Continuous Training Course for Interpreters and Translators of the Administration". Lingua Legis 27: 11-31. 